\title{
Relé em Malha Fechada Robusto à Distúrbios após Filtragem Pente
}

\author{
Pedro I. C. Martins. ${ }^{*}$ Francisco V. E. Lemos. ${ }^{*}$ \\ Otacilio da M. Almeida. ${ }^{* *}$ José M. P. de Menezes, Jr** \\ * Programa de Pós-Graduação em Engenharia Elétrica, UFPI, PI \\ (e-mail: pjago@protonmail.com; victorlemos@ufpi.edu.br) \\ ** Departamento de Engenharia Elétrica, UFPI, PI \\ (e-mail: (otacilio; josemenezesjr)@ufpi.edu.br)
}

\begin{abstract}
The relay method, since it was presented, had wide acceptance in the industry, due to its practicality and performance. However, the classical method of relay identification is subject to problems, such as errors arising from continuous disturbances in the process under analysis. In this work is presented a proposal of improvement in the stability of the relay with hysteresis, applying a comb filter. This filter is intended to select only the odd harmonic range. A level tank was used for the identification, in which the classical and the proposed method was applied. Identification by the relay method with hysteresis using comb filter was close to that obtained by the method without the filter by $5 \%$, with the advantage of being more robust.

Resumo: O método do relé, desde que foi apresentado, teve ampla aceitação na indústria, devido sua praticidade e desempenho. Contudo, o método clássico de identificação por relé está sujeito a problemas, como erros oriundos de distúrbios contínuos no processo em análise. Neste trabalho é apresentada uma proposta de melhoria na estabilidade do relé com histerese, aplicando um filtro pente. Este filtro tem como objetivo selecionar apenas a faixa de harmônicas ímpares. Foi utilizado um tanque de nível para a identificação, no qual foi aplicado o método clássico e o proposto. A identificação pelo método do relé com histerese usando filtro pente foi próxima aquela obtida pelo método sem o filtro por $5 \%$, com a vantagem de ser mais robusta.
\end{abstract}

Keywords: Auto-tuning. Comb Filter. Identification. Relay with hysteresis.

Palavras-chaves: Auto-tuning. Filtro Pente. Identificação. Relé com histerese.

\section{INTRODUÇÃO}

O relé de auto-sintonia, desde que foi introduzido na década de 80 por Åström and Hägglund (1984), como uma alternativa rápida e simples de sintonia dos controladores PID, foi amplamente adotado na indústria Berner et al. (2016). A priori, não necessita de qualquer conhecimento prévio sobre o processo em foco, além de operar em realimentação junto com a malha do processo.

Para superar o problema de assimetria do experimento do relé, em (Park et al., 1997) é apresentada uma correção do relé medindo a variação dos tempos de chaveamento e do ganho na sua frequência, para deslocar o bias de entrada. Um relé com dois filtros é proposto por Lee et al. (2011) para superar os problemas causados por deslizamento na saída. Um filtro de baixa frequência é usado para remover ruídos e um filtro de alta frequência é usado para remover desvios de baixa frequência. Esta abordagem visou alcançar um compromisso entre a rejeição de perturbações de baixa e alta frequência, enquanto se obtém um ganho próximo a um, em uma largura de banda ampla.

Goodwin et al. (2018) apresentaram um relé com o uso de um filtro ressonante para um sistema de grandes perturbações em uma larga faixa de frequências. O filtro ressonante projetado, possui ganho unitário em uma frequência $\omega$ e ganho muito pequeno em qualquer outro valor. Em relação a escolha do filtro de Goodwin et al. (2018), é possível optar por diferentes tipos. Caso se compare com o filtro usado em Lee et al. (2011), também de segunda ordem, o filtro $\mathrm{F}(\mathrm{s})$ fornece uma capacidade maior para a rejeição de perturbações. O filtro altamente ressonante, $\mathrm{F}(\mathrm{s})$, não apresenta uma largura de banda ampla, devido à sua seletividade de frequência e capacidades inerentes de mudança de fase. Como desvantagem desta proposta de Goodwin et al. (2018) é que o filtro pode desviar a frequência de oscilação da frequência crítica.

Em seu trabalho, da Silva and Barros (2017) propõem uma estrutura do relé realimentado para superar as limitações da oscilação estável sob grandes distúrbios ou deslizamento. Esta estrutura é composta de um bloco para remover a perturbação estática e deslizamento, seguido por um relé. O bloco consiste em um filtro passa-altas simples, seguido por outro relé idêntico, e então um integrador, para remover a sensibilidade a alta frequência. A estrutura é robusta a distúrbios de amplitude bem maior do que a do relé, ao custo de uma pequena modificação na função descritiva original, por influência dos filtros.

Nesse contexto, propõe-se a utilização de um filtro pente, que seleciona apenas as frequências harmônicas aquela 
identificada pelo relé com histerese, de maneira adaptativa. $\mathrm{Na}$ forma subtrativa, o filtro ainda é capaz de remover a oscilação na saída específica ao relé, e proporcionar uma variável de controle filtrada, portanto, adequada para a sintonia automática em paralelo à atuação de controle.

Este trabalho é organizado da seguinte maneira: na Seção 2 a estrutura proposta para se adaptar à distúrbios do tipo degrau é discutida; na Seção 3 a estrutura proposta para se adaptar à distúrbios do tipo rampa é discutida; na Seção 4 é apresentada a planta teste, com os resultados experimentais e discussão a cerca deles e na seção 5 é feita a conclusão.

\section{ESTRUTURA TIPO 0}

Nesta Seção, um exemplo simples de adaptação à um erro do tipo degrau é analisado. Classifica-se o filtro suficiente como tipo 0, e apresenta-se a sua estrutura de adaptação. Por comparação, aplica-se o relé com histerese sem filtro, da Figura 1. O sistema tomado como exemplo foi um cooler de cpu, com modelo de função de transferência (1).

$$
G(z)=\frac{0.3104 z^{-2}+0.0376^{-3}}{1-1.151 z^{-1}+0.3052^{-2}}
$$

O período de amostragem $T$ foi $0.25 \mathrm{~s}$, e a variável de saída $y$ foi o número de pulsos do enconder em cada período. A variável de controle $u$, proporcional à tensão no motor, foi dada em porcentagem. Fixou-se a variável de referência $r$ inicialmente em 100. Alimenta-se o erro $e=r-y$ ao relé.

\subsection{Relé comum com erro degrau}

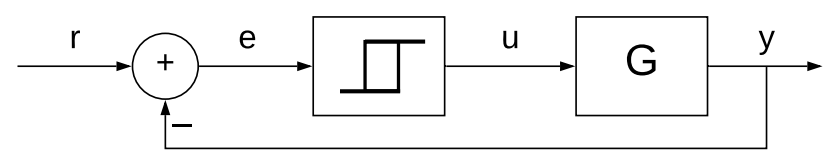

Figura 1. Relé com histerese em malha fechada

Aplica-se ao cooler o relé com histerese em malha fechada, configurado com amplitude $d=25$, bias $b=50$, e histerese $h=5$. Na Figura 2 apresenta-se a sua resposta em $u, y, r$; onde o eixo horizontal é a numeração da amostra. Nota-se que a amplitude de oscilação em $y$ foi $a=22.5$, e o período do ciclo final foi $n_{c}=9$, em quantidade de amostras.

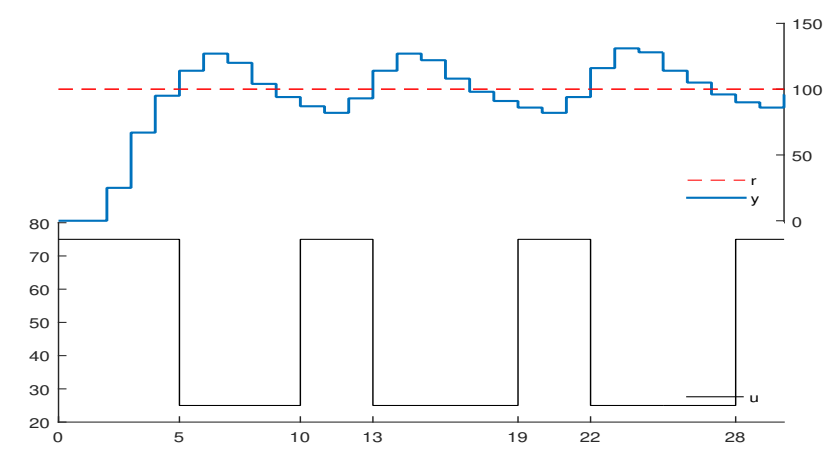

Figura 2. Resposta assimétrica ao relé com histerese

Através da função descritiva do relé, definida em (2), é possível aproximar o ganho e a fase do sistema para a frequência $\omega_{c}=2 \pi / n_{c}$. Tal aproximação degrada quando, o relé introduz componentes harmônicas perceptíveis em $y$, ou ainda, a atuação $u$ do relé tem períodos assimétricos.

$$
N\left(j \omega_{c}\right)=\frac{4 d}{\pi a}\left(\sqrt{1-(h / a)^{2}}-j h / a\right)
$$

Na Figura 2, nota-se que o semiciclo positivo dura 3 amostras, e o semiciclo negativo dura 6 amostras. Para corrigir tal assimetria seguindo a estratégia de Shen et al. (1996), é necessário diminuir o bias do relé para $b=$ $100 / G(1)$. Em contraste, na estrutura proposta, opta-se por adaptar a referência $r$ à saída $y$, admitindo variações menores do que a sua amplitude $a$.

\subsection{Relé com filtro pente com erro degrau}

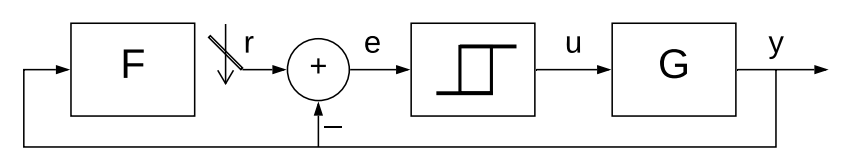

Figura 3. Estrutura tipo 0 ativada após pré-tuning

A estrutura proposta para eliminar o erro, do tipo degrau, é a realimentação de $y$ em $r$ através do filtro pente definido em (3). O filtro é adaptado pela duração $n_{s}$ em amostras do semi-ciclo anterior, ou seja, atualiza-se $n_{s}$ com a contagem definida entre cada transição do relé. Para determinar o valor inicial de $n_{s}$, e posicionar $y$ próximo ao seu valor médio, reserva-se uma fase de pré-tuning no primeiro ciclo, no qual o sistema é acionado por um relé comum. Uma idéia semelhante já foi mencionada por Astrom (1995), apesar de não especificar o tipo de filtro.

$$
F_{0}(z)=\frac{1+z^{-n_{s}}}{2}
$$

A resposta é apresentada na Figura 4 . O filtro $F_{0}$ realiza, em cada momento, uma média entre o semi-ciclo atual e o semi-ciclo anterior. No semi-ciclo após pré-tuning, de amostras 13 à 17, nota-se que $n_{s}$ foi adaptado para 3 , a duração do semi-ciclo anterior; nessa situação, o desvio positivo atual não é cancelado e média realizada em $F_{0}$ aumenta, prolongando o semi-ciclo positivo subsequente. No terceiro ciclo, de amostras 21 à 29, nota-se que y já possui uma resposta simétrica, e o desvio em $y$ cancela-se à cada momento com o seu desvio no semi-ciclo anterior.

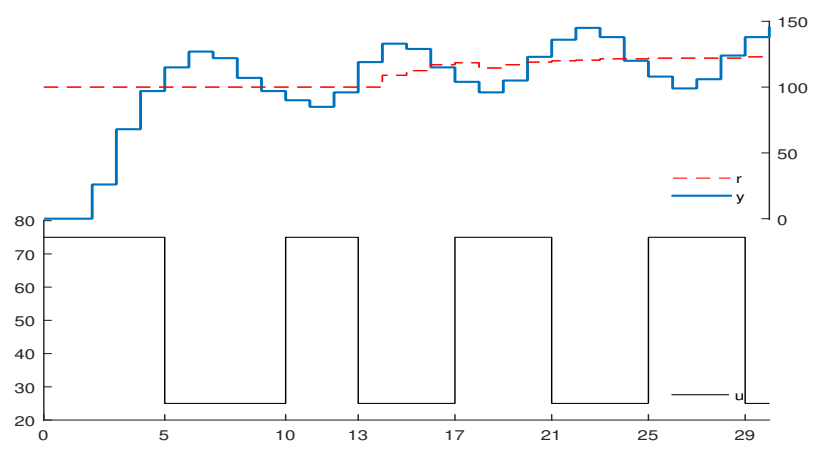

Figura 4. Resposta simétrica ao relé com histerese e filtro

Nas frequências harmônicas pares de $\omega_{c}$, assimétricas, o filtro pente tem ganho unitário e defasagem nula. Por outro 
lado, nas frequências harmônicas ímpares, simétricas, o ganho é próximo à zero. Assim, o filtro pente complementa o relé simétrico de maneira notável, pois a realimentação do erro destaca apenas as oscilações harmônicas ímpares, e assim rejeita assimetrias ou pequenas variações de $n_{s}$.

Em contraste à alternativa de da Silva and Barros (2017), o filtro proposto não altera a função descritiva do relé, logo (2) continua aplicável. O filtro de Goodwin et al. (2018) seleciona apenas a frequência ressonante $\omega_{c}$, e portanto perde a informação das frequências ímpares. Por outro lado, o filtro pente proposto com uma pequena histerese, mostra-se bem apropiado para a identificação de frequências próximas a frequência crítica, onde $n_{s}$ geralmente é pequeno.

\section{ESTRUTURA TIPO 1}

Nesta Seção, o desafio da adaptação ao erro do tipo rampa é analisado. Classifica-se o filtro suficiente como tipo 1, e o elabora a partir do conceito de média estabelecido para o filtro 0. Adiciona-se um mecanismo de correção à falhas e assimetria. A estrutura tipo 1 é relevante para sistemas com integrador, onde um distúrbio estático na entrada provoca uma rampa na saída. Na Figura 5 ilustrase o distúrbio negativo $-v$ paralelo à ação de controle. Os parâmetros $a_{s}$ e $n_{s}$ do fitro final são adaptados nos momentos de transição do relé.

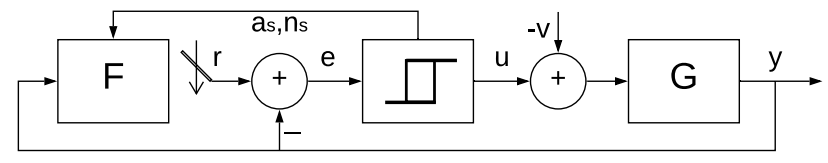

Figura 5. Estrutura tipo 1 com pré-tuning e correção ativa

\subsection{Filtro pente tipo 1}

Para se obter um filtro pente do tipo 1, com erro nulo para para o sinal rampa, é necessário que a função de transferência $\frac{E_{1}(z)}{Y_{1}(z)}$ tenha numerador com dois pólos em -1. Além disso, como o objetivo é filtrar as harmônicas pares, o numerador deve ser um polinômio de ordem múltipla da duração de semi-ciclo, $n_{s}$. Dessa forma, é suficiente escolher o numerador de ordem $n_{c}=2 n_{s}$, resultante da repetição da função de transferência $\frac{E_{0}(z)}{Y_{0}(z)}$ do tipo 0:

$$
\begin{aligned}
E_{0} / Y_{0}(z) & =F_{0}(z)-1=-\frac{1-z^{-n_{s}}}{2} \\
E_{1} / Y_{1}(z) & =-\left(\frac{E_{0}(z)}{Y_{0}(z)}\right)^{2}=-\frac{z^{-n_{c}}-2 z^{n_{s}}+1}{4}, \\
F_{1}(z) & =\frac{E_{1}(z)}{Y_{1}(z)}+1=\frac{3+2 z^{n_{s}}-z^{-n_{c}}}{4} .
\end{aligned}
$$

Extrapolando o sistema de equações (4) para o tipo 2, é possível ainda desenvolver um filtro pente com erro de regime nulo para uma aceleração constante. Todavia, a ordem do filtro aumenta para $3 n_{s}$, junto com a memória necessária para a sua implementação digital. Ainda assim, para $n_{c}$ próximo ao período crítico, é razoável que se armazene a resposta $y$ ao relé durante um ciclo completo, o que é suficiente para o filtro tipo 1 .

\subsection{Correção ativa contra falhas}

Os filtros pente utilizados possuem uma certa sensibilidade na primeira oscilação, quando os valores finais de $n_{s}$ e $n_{c}$ ainda não foram estabelecidos. Além da fase de pré-tuning, convém corrigir ativamente a saída do filtro quando o semiciclo atual é maior do que o semi-ciclo anterior. Nessa condição, os valores com atraso do filtro são congelados, ou seja, deixam de ser atualizados. Para um sistema de primeira ordem, essa condição corresponde aproximadamente aos valores de máximo ou mínimo anterior, dependendo da multiplicidade em $n_{s}$ e da assimetria dos ciclos anteriores. Dessa forma, evita-se que $r$ siga $y$ exatamente, e que a entrada $e$ do relé não alcance o valor de histerese.

\subsection{Correção da assimetria}

Na condição de falha estabelecida, convém ainda corrigir a assimetria presente no último ciclo, através do parâmetro de assimetria $a_{s}$. A adaptação do filtro nesse caso resulta na implementação (5). Fora da condição de falha, o parâmetro $a_{s}$ é setado igual à 1 , e o filtro funciona diretamente. No momento em que a falha é detectada, os valores $y_{k-n_{s}} \mathrm{e}$ $y_{k-n_{c}}$ são fixados nos seus valores atuais, representando os limites da última oscilação. Ao mesmo tempo, o parâmetro $a_{s}$ é atualizado com a razão dos semi-ciclos $\frac{n_{s}}{n_{c}-n_{s}}$, e o termo atual $y_{k}$ variável é então ponderado por $y_{k-n_{c}}$, de forma a atrasar ainda mais a atualização do filtro.

$$
r_{k}=\frac{3\left(a_{s} y_{k}+\left(1-a_{s}\right) y_{k-n_{c}}\right)+2 y_{k-n_{s}}-y_{k-n_{c}}}{4}
$$

Para o sistema sem integrador utilizado na Seção 2, notase que os mecanismos de correção contra falha e assimetria não foram necessários, e apenas o ciclo de pré-tuning já foi suficiente. Um fator que os mecanismos apresentados não corrigem é a ocorrência de um alto distúrbio degrau na saída do sistema, de magnitude maior do que a amplitude de oscilação de $y$. Nessa situação, de acordo com o instante em que o distúrbio ocorre dentro do semi-ciclo, é possível que o valor de $r$ estabilize próximo a $y$, o suficiente para que o erro seja menor do que a histerese. Todavia, no sistema integrador em objetivo, que segue, os mecanismos foram suficientes para a boa aplicação do filtro.

\section{RESULTADOS}

Para avaliar a robustez da estrutura 1 à distúrbios rampa, utilizou-se a planta MPS-PA da FESTO, Figura 6, um sistema didático de aprendizagem para processos industriais (HELMICH, 2008). Especificamente, utilizou-se o processo de nível, no qual água é transportada entre o reservatório $\mathrm{B} 102$ e o reservatório B101, em diferentes alturas. O nível no tanque superior B102 é controlado por meio da bomba P101, localizada na base, e do sensor ultrassônico LIC B101, localizado no teto. A válvula manual V110, que conecta ao tanque inferior B101, foi semi-aberta para reproduzir o distúrbio rampa na altura do nível.

Para se obter os resultados no tanque de nível, o período de amostragem $T$ foi $1 \mathrm{~s}$. A variável de saída $y$ foi a tensão do sensor entre 0 e $10 \mathrm{~V}$, proporcional ao nível. A variável de controle $u$ foi o sinal proporcional à tensão da bomba, de 0 a $10 \mathrm{~V}$. Fixou-se a referência $r$ inicialmente em $4.0 \mathrm{~V}$. O 


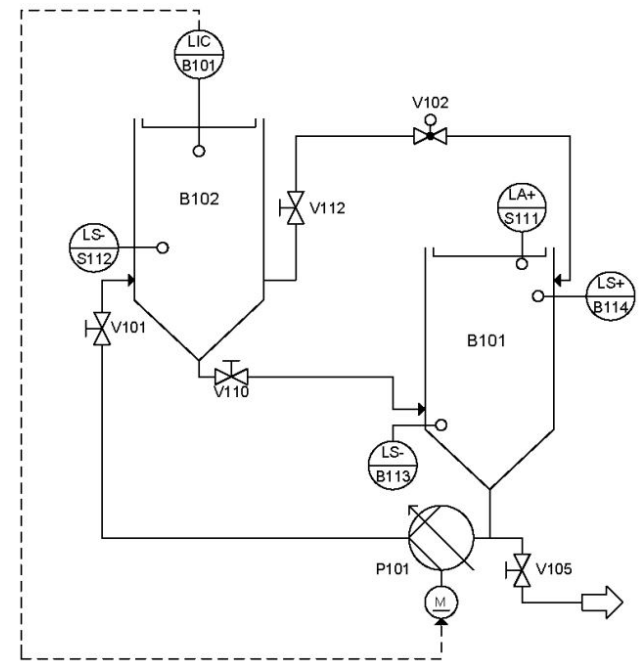

Figura 6. Sistema do Processo de Nível.

relé foi configurado com amplitude $d=2.5 \mathrm{~V}$, bias $b=5.0$ $\mathrm{V}$, e histerese $h=0.1 \mathrm{~V}$. O distúrbio $v$ foi quase estático. O modelo aproximado para o tanque foi $G(s)=\frac{K}{s}$.

\subsection{Relé comum com distúrbio rampa}

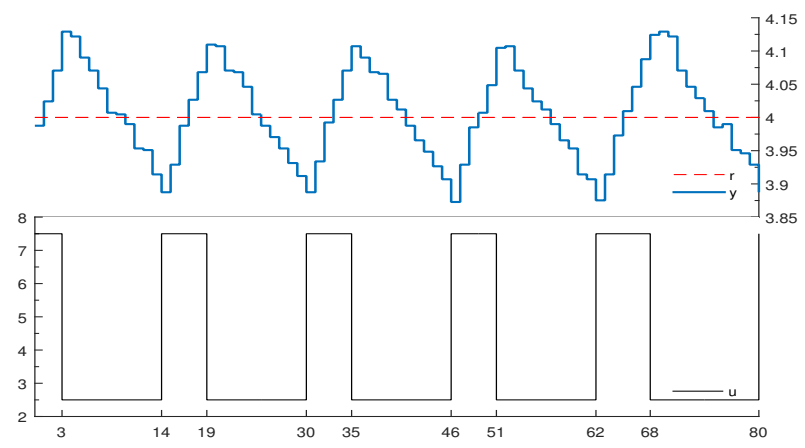

Figura 7. Resposta assimétrica ao relé com histerese

Na Figura 7, apresenta-se a resposta assimétrica do relé com histerese. Nessa situação, o valor efetivo de $u$ equilibra o distúrbio $v$ provocado pela válvula; que pode ser calculado em (6), no ciclo de 46 à $62 \mathrm{~s}$, onde o tempo alto foi $t_{\text {on }}=5 \mathrm{~s}$, e o tempo baixo foi $t_{\text {off }}=11 \mathrm{~s}$.

$$
v=\frac{t_{\mathrm{on}}(b+d)+t_{\mathrm{off}}(b-d)}{t_{\mathrm{on}}+t_{\mathrm{off}}}=4.0625 \mathrm{~V}
$$

Com o distúrbio $v$ estimado, e calculando-se a integral do erro $I_{e}$ no período, é possível ainda identificar $K$ por (7), fórmula esta adaptada de Berner et al. (2016) para sistemas do tipo integrador com atraso no tempo.

$$
\begin{aligned}
K & =\frac{2 h}{(b+d-v) t_{\mathrm{on}}}-\frac{I_{e}}{(b-v) t_{\mathrm{on}} t_{\mathrm{off}}} \\
& =\frac{0.2}{17.187}-\frac{0.1897}{51.562}=0.007958 \mathrm{~V} / \mathrm{s}
\end{aligned}
$$

\subsection{Relé com filtro pente com distúrbio rampa}

Na Figura 8, o relé com filtro tem chaveamento é simétrico e portanto não mais equilibra o distúrbio $v$. Identificado $v$ no ciclo pré-tuning aplicando (6), é possível aplicar outra estratégia e identificar $K$, agora pela inclinação $m$ da reta de subida filtrada em $r$, aplicando (8).

$$
K=\frac{m}{b-v}=\frac{0.009327}{5.0-4.0625}=0.009949 \mathrm{~V} / \mathrm{s}
$$

A estratégia com o filtro calculou um $K 25 \%$ maior do que a identificação a relé convencional. Vale notar porém que o valor de $v$ aumenta ligeiramente a medida que a coluna de água sobre a válvula aumenta.

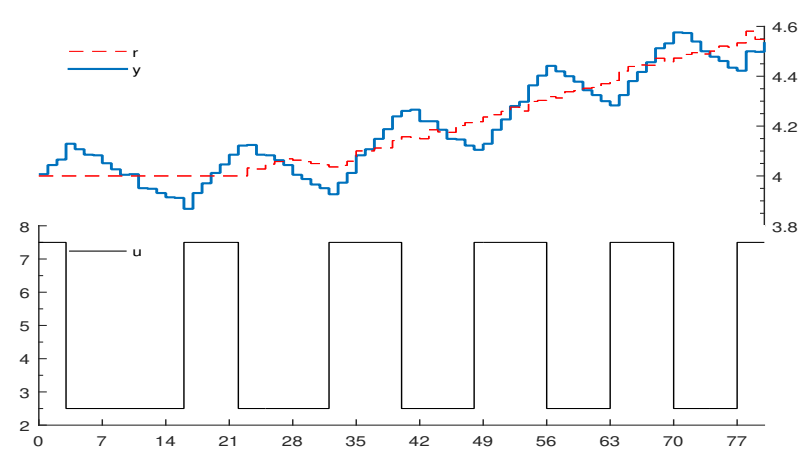

Figura 8. Resposta simétrica ao relé com histerese e filtro

Na Figura 9 apresenta-se as curvas de erro de cada experimento demarcadas pelo chavemento dos seus relés. A estratégia de identificação (7) também pode ser aplicada na variável $e$ filtrada, com a simplificação de que nesse caso $t_{\mathrm{on}}=t_{\mathrm{off}}=7 \mathrm{~s}$. $\mathrm{O}$ valor de $I_{e}$ foi considerado zero. $\mathrm{O}$ resultado é $K=0.0083116$, menos de $5 \%$ maior do que a estimativa do relé com histerese sem filtro.

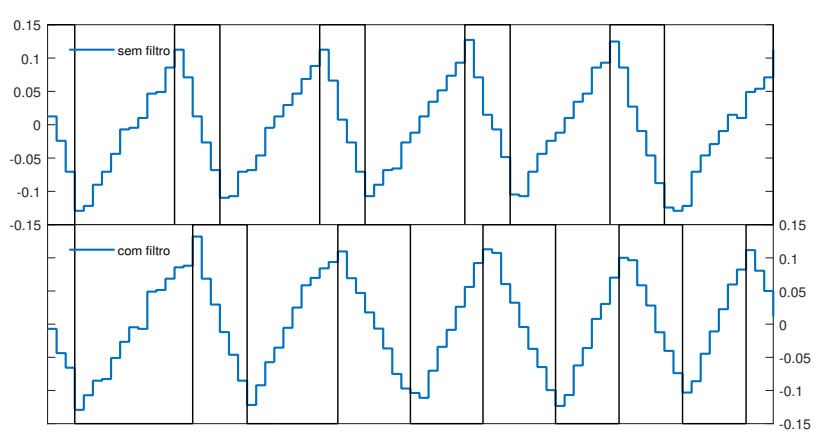

Figura 9. Erro - Entrada do relé com e sem o filtro

Na curva de erro correspondente ao teste com filtro, é interessante notar a ação dos mecanismos de correção de falha e assimetria durante o segundo semi-ciclo negativo, logo após a fase de pré-tuning. Apesar do aumento provocado na rampa em $y$, o erro $e$ manteve-se razoavelmente com a mesma inclinação em função da correção apresentada.

\section{CONCLUSÃO}

Avaliou-se a utilização de filtros da classe pente para a manutenção de oscilações simétricas do relé com histerese em sistemas de baixa ordem. Observou-se que o filtro pente é capaz de remover distúrbios do tipo degrau e rampa da entrada do relé, e alimentar apenas as frequências harmônicas ímpares correspondentes à sua atuação. Com 
a estrutura proposta, identificou-se um sistema de nível do tipo integrador e com uma variação de $5 \%$ em relação ao método do relé assimétrico convencional.

\section{AGRADECIMENTOS}

Os autores agradecem ao Programa de Pós-Graduação em Engenharia Elétrica da Universidade Federal do Piauí. Assim como ao GRASI - UFPI e ao Laboratório de Controle, Automação e Telecomunicações - UFPI pela disponibilização dos equipamentos.

\section{REFERÊNCIAS}

Åström, K.J. and Hägglund, T. (1984). Automatic tuning of simple regulators with specifications on phase and amplitude margins. Automatica, 20(5), 645-651.

Astrom, Karl J. e Hagglund, T. (1995). PID Controllers: Theory, Design, and Tuning. Instrument Society of America, 2 edition.

Berner, J., Hägglund, T., and Åström, K.J. (2016). Asymmetric relay autotuning-practical features for industrial use. Control Engineering Practice, 54, 231-245.

da Silva, M.T. and Barros, P.R. (2017). Application of a robust to disturbance relay feedback for identification of a grain dryer. XIII, Simpósio Brasileiro de Automaçao Inteligente, SBAI.

Goodwin, G.C., Seron, M.M., and Townsend, C. (2018). A modified relay autotuner for systems having large broadband disturbances. Automatica, 94, 178-185.

HELMICH, J. (2008). Manual da festo.

Lee, J., Kim, J.S., Byeon, J., Sung, S.W., and Edgar, T.F. (2011). Relay feedback identification for processes under drift and noisy environments. AIChE Journal, 57(7), 1809-1816.

Shen, S.H., Wu, J.S., and Yu, C.C. (1996). Use of biasedrelay feedback for system identification. AIChE Journal, 42(4), 1174-1180. 